\title{
Giant adrenal hemangioma: Unusual cause of huge abdominal mass
}

\author{
Mohamed Tarchouli, MD; ${ }^{*}$ Adil Boudhas, MD; ${ }^{\dagger}$ Moulay Brahim Ratbi, MD; ${ }^{*}$ Mohamed Essarghini, MD; \\ Noureddine Njoumi, MD; Khalid Sair, MD; ${ }^{*}$ Aziz Zentar, $M D^{\S}$
}

*Department of Digestive Surgery I, Mohammed V Military Hospital, Faculty of Medicine and Pharmacy, Mohammed V University, Rabat, Morocco; 'Department of Pathology, Mohammed V Military Hospital, Faculty of Medicine and Pharmacy, Mohammed V University, Rabat, Morocco; §Department of Digestive Surgery II, Mohammed V Military Hospital, Faculty of Medicine and Pharmacy, Mohammed V University, Rabat, Morocco

Cite as: Can Urol Assoc J 2015;9(11-12):E834-6. http://dx.doi.org/10.5489/cuai.2967 Published online November 4, 2015.

\section{Abstract}

Adrenal hemangioma is an extremely rare benign and non-functioning neoplasm of the adrenal gland. We report a case of a 71-year-old woman admitted for intermittent abdominal pain and abdominal distension associated with vomiting and chronic constipation for 5 years. Physical examination revealed a large abdominal mass. Both computed tomography scan and magnetic resonance imaging suggested hemangioma in the right lobe of the liver. Laboratory examinations and tumour markers were within normal limits, except for hypochromic microcytic anemia. The mass was removed intact by conventional surgery and histopathology revealed a cavernous hemangioma of the adrenal gland with no signs of malignancy. Surgical resection was curative, with no recurrence at the 2-year follow-up.

\section{Introduction}

Adrenal hemangiomas are extremely rare and frequently discovered incidentally in asymptomatic patients during imaging studies. Their differential diagnosis with adrenal malignancies is rather challenging because masses are usually large and radiographic aspects are not always specific. Most are diagnosed after surgery with a histological examination. We report an interesting case of a large adrenal hemangioma in a patient with a bulky abdominal mass.

\section{Case report}

A 71-year-old women being treated for hypertension, complained of intermittent abdominal pain and steadily increasing abdominal girth for 5 years. She also experienced vomiting and chronic constipation, without jaundice, fever or weight loss. Physical examination revealed a large mass in the right side of the abdomen. Abdominal computed tomography (CT) demonstrated a very large, hypoattenuated and well-circumscribed hepatic mass with multiple calcifications and faint marginal enhancement on the post-contrast study. This mass involved the entire right lobe of the liver causing displacement and compression of the right kidney, portal vein and vena cava. There were no areas of necrosis and thrombosis, and no evidence of deep lymph nodes (Fig. 1). Magnetic resonance imaging (MRI) was performed showing a wellencapsulated lobulated mass measuring $35 \times 26 \times 22 \mathrm{~cm}$, with a heterogeneous internal structure containing cystic areas and an irregular peripheral enhancement on the postcontrast images (Fig. 2). Both CT scan and MRI suggested a diagnosis of hemangioma in the right lobe of the liver. In addition, laboratory examinations (including blood cell count, liver function tests, lipid parameters and serology tests for hepatitis B and C) were normal, apart from a hypochromic microcytic anemia (hemoglobin $8.4 \mathrm{~g} / \mathrm{dL}$ ). Tumour markers, including alpha-fetoprotein, carcinoembryonic antigen and carbohydrate antigen (CA 19.9 and CA 125), were within normal limits. Also, functional investigations concerning blood cortisol, dexamethasone suppression test and urinary catecholamines on 24-hours collection were normal.

Based on these findings, a malignancy could not be excluded and surgical resection was mandatory. Through a large abdominal midline incision, we discovered a huge retroperitoneal tumour occupying the entire right side of the abdominal cavity and displacing the liver to the left. This tumour, easily releasable from the surrounding structures, appeared to come from the adrenal gland with a vascular pedicle (Fig. 3). The tumour was removed intact and the pathological examination revealed a smoothly and completely encapsulated mass measuring $42 \times 38 \times 17 \mathrm{~cm}$ with mixed cystic-solid content (Fig. 4). Microscopically, extensive necrotic and hemorrhagic areas were present with dilated vascular spaces bordered by an endothelial proliferation (Fig. 5). Normal adrenal gland parenchyma was observed on the surface of the mass. These findings are typical of a cavernous hemangioma of the adrenal gland. The patient was discharged after an uneventful postoperative period. No biological or radiographic follow-up was necessary regarding the benign pathology. Surgical resection was curative, with good outcomes at the 2-year follow-up. 


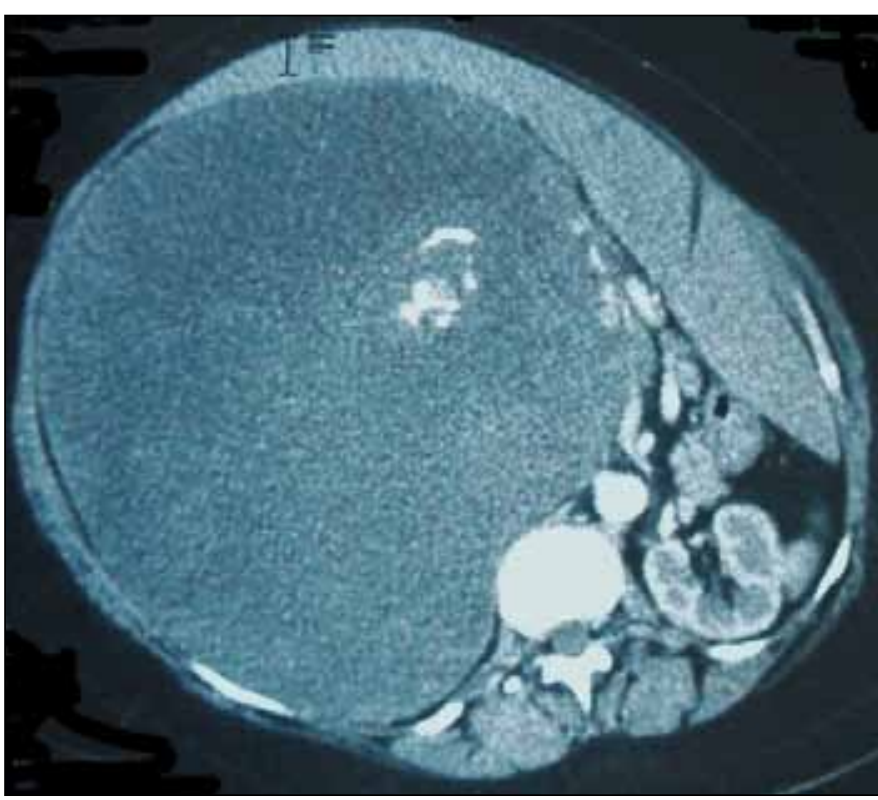

Fig. 1. Axial computed tomography scan section demonstrating a voluminous well-circumscribed mass with multiple calcifications, heterogeneous content and compression of surrounding structures.

\section{Discussion}

Adrenal hemangiomas are benign neoplasms arising from endothelial cells that line blood vessels. Since the first surgically excised case in 1955, about 65 adrenal hemangiomas have been reported. ${ }^{1,2}$ They are usually unilateral and non-functional tumours. Affected individuals are frequently between 50 and 70 years of age, with a 2:1 female-to-male ratio. Tumour size ranges from 2 to $25 \mathrm{~cm}$ in diameter, with most measuring more than $10 \mathrm{~cm}$. These tumours are most commonly asymptomatic and incidentally discovered during imaging studies performed for an unrelated reason

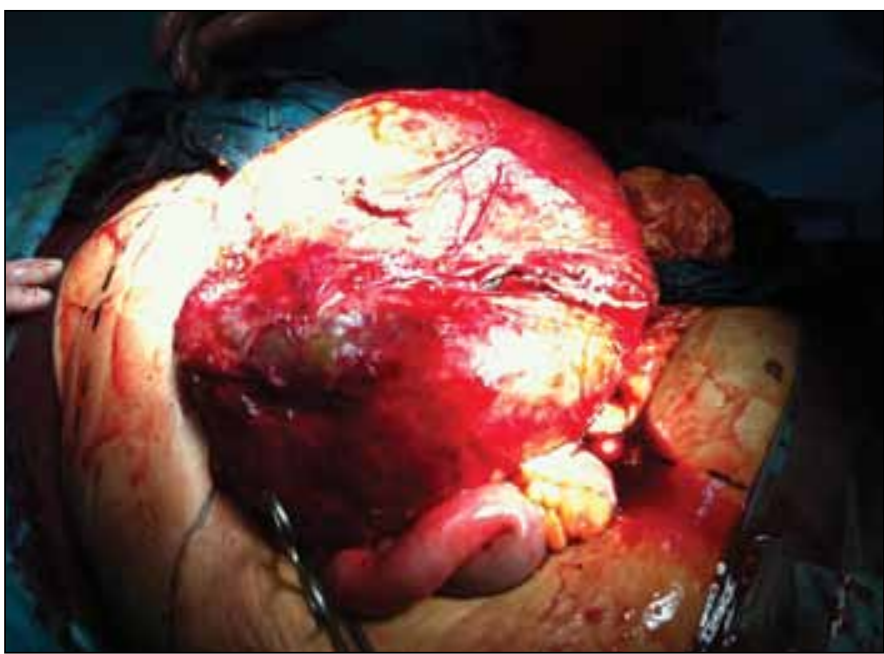

Fig. 3. Intraoperative view.

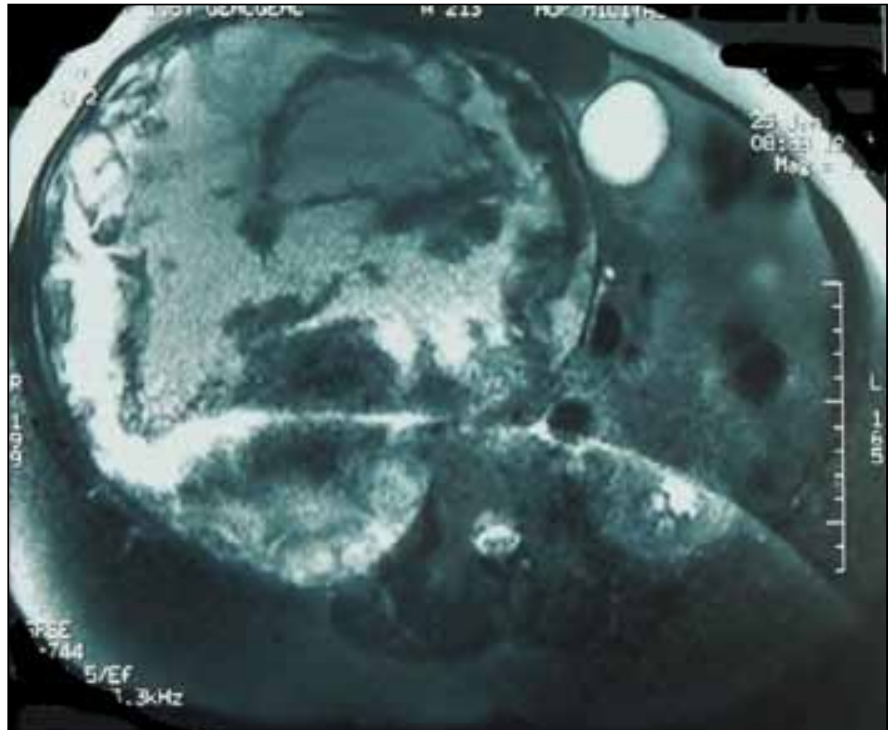

Fig. 2. Magnetic resonance imaging transverse section (contrast-enhanced T2weighted image) revealing a high-signal intensity mass with a heterogeneous internal structure containing cystic areas and irregular peripheral enhancement.

(i.e., incidentaloma). Symptomatic patients may present with abdominal pain or mass-related symptoms secondary to compression of surrounding structures. In our case, the lesion measured $42 \mathrm{~cm}$ in its longest diameter; we believe this is the largest adrenal hemangioma reported to date.

Histologically, adrenal hemangiomas are usually cavernous and rarely capillary in type. The lesions are well-encapsulated and located in the adrenal cortex. The presence of multiple dilated vascular spaces at the periphery is an important feature, whereas complex modifications involve necrosis, calcifications, fibrosis, thrombosis, and hemorrhage.

Typical findings of adrenal hemangioma on CT include a heterogeneous and hypodense lesion with some calcifica-

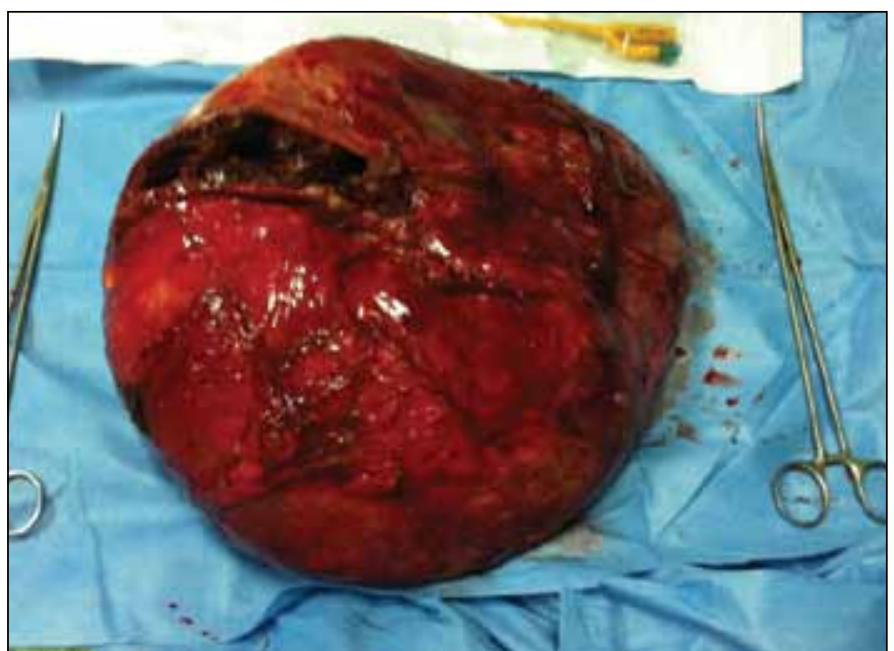

Fig. 4. Gross appearance of resected specimen showing a voluminous welldelineated mass measuring about $42 \mathrm{~cm}$ in diameter. 


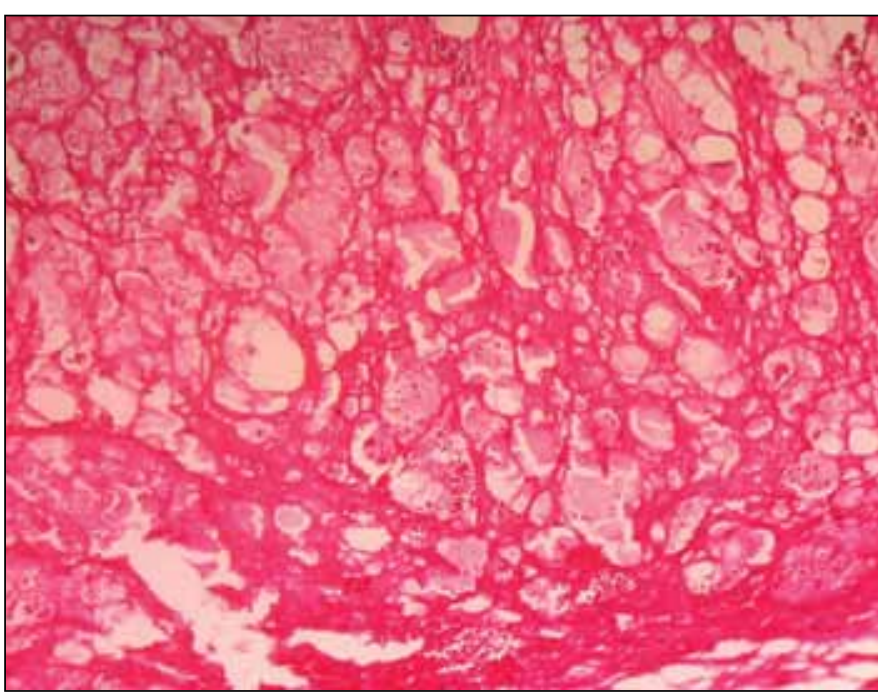

Fig. 5. Histopathological findings of resected specimen with microscopic image showing multiple dilated vascular spaces lined by a single layer of mature endothelial cells (hematoxylin and eosin staining, $\times 100$ ).

tions, as was seen in our patient. Post-contrast CT displays a characteristic peripheral irregular enhancement with progression from the periphery to the center of the tumour. This centripetal enhancement pattern, less frequent in hepatic hemangiomas, is highly specific for hemangioma and is not seen in other adrenal tumours. In addition, calcifications are attributed to multiple phleboliths inside the dilated vascular spaces and characteristically appear speckled throughout the entire mass. However, they lack specificity because they can be encountered in other adrenal tumours, such as pheochromocytoma, carcinoma and adenoma..$^{3-5}$ The MRI reveals a hypointense, heterogeneous lesion with central hyper-intensity representing hemorrhage on T1-weighted sequences, and high signal intensity in T2-weighted sequences. Late peripheral enhancement occurs after the administration of gadolinium. However, these findings can also be observed in other adrenal tumours and are not pathognomonic of adrenal hemangioma. ${ }^{6}$

Surgical excision remains mandatory for large adrenal masses exceeding $4 \mathrm{~cm}$ in size due to the impossibility of excluding malignancy. Additionally, surgical treatment is required for relieving pressure-related symptoms, precluding complications, such as spontaneous hemorrhage, and providing a definitive diagnosis after pathological examination of the excised mass. ${ }^{4,7}$ Several open techniques, including transabdominal, flank, and posterior approaches, have been described. Although laparoscopic adrenalectomy is standard procedure for small adrenal masses, it is still contraindicated for giant adrenal masses. ${ }^{1,8}$ In our patient, the adrenal mass was removed with open transabdominal surgery due to the size of the tumour. However asymptomatic patients with adrenal masses smaller than $4 \mathrm{~cm}$ may be conservatively managed by regular follow-up imaging.

\section{Conclusion}

Although rare, adrenal hemangioma should be included in the differential diagnosis of adrenal, renal and right-lobe liver tumours, especially when radiographic signs are suggestive for this diagnosis. Surgical excision is mostly required to rule out malignant disease and preclude spontaneous rupture particularly in large size tumours. Patients with large adrenal lesions, like the case presented, are preferentially treated with open surgery.

Competing interests: The authors declare no competing financial or personal interests.

This paper has been peer-reviewed.

\section{References}

1. Noh JJ, Choi SH, Hwang HK, et al. Adrenal cavernous hemangioma: A case report with review of the literature. JOP 2014;15:254-7. http:// dx.doi.org/10.6092/1590-8577/2402.

2. Wang L, Dang Y, He R, et al. Rare cavernous hemangioma of adrenal gland: Case report. Sao Paulo Med J 2014;132:249-52.

3. Abou El-Ghar M, Refaie H, El-Hefnawy A, et al. Adrenal hemangioma: Findings at multidetector CT with short review of the literature. Case Rep Radiol 2011;2011:601803. http:// dx.doi. org/10.1155/2011/601803.

4. Kieger AJ, Nikolaidis P, Casalino DD. Adrenal gland hemangioma. J Urol 201 1;186:2415-6. http:// dx.doi.org/10.1016/i.juro.2011.09.067.

5. Quildrian SD, Silberman EA, Vigovich FA, et al. Giant cavernous hemangioma of the adrenal gland. Int J Surg Case Rep 2013;4:219-21. http://dx.doi.org/10.1016/i.i.jscr.2012.11.022.

6. Harzallah L, Zouari L, Ben Chérifa L, et al. Hémangiome surrénalien: à propos d'un cas. Ann Endocrinol 2006;67:624-7. http://dx.doi.org/10.1016/S0003-4266(06)73018-9.

7. Alhairi K, Alhasan I, Alzerwi N, et al. Adrenal haemangioma. BMJ Case Rep 2011;2011. http:// dx.doi. org/10.1136/bcr.12.2010.3604.

8. Edwards JP, Stuart HC, Urbanski SJ, et al. A rare cavernous hemangioma of the adrenal gland. Int I Surg Case Rep 2014;5:52-5. http://dx.doi.org/10.1016/i.i.jscr.2013.11.003.

Correspondence: Dr. Mohamed Tarchouli, Department of Digestive Surgery I, Mohammed V Military Hospital, Rabat, Morocco; mtarchouli@gmail.com 